\title{
Ultra Sonographic Mirror Depiction of Enormous Bizarre Intramural Myomas - Laparoscopic Dissection
}

\author{
Sofoudis Chrisostomos ${ }^{1 *}$, Dedoulis Evangelos ${ }^{2}$ and Zioris Konstantinos ${ }^{2}$ \\ ${ }^{1}$ Department of Obstetrics and Gynaecology, Konstandopoulio General Hospital, Greece \\ 2Mitera, Maternal Hospital, Greece
}

*Corresponding author: Chrisostomos Sofoudis, Department of Obstetrics and Gynaecology, Konstandopoulio General Hospital, Greece.

Received Date: March 20, 2020

Published Date: May 11, 2020

\begin{abstract}
Leiomyomas represent the most common type of benign tumours of the female genital tract. Assiduous preoperative imaging findings reflect proper therapeutic mapping. In cases of female patients of reproductive age, the ultimate goal remains the fertility preservation and the quality of life of the patient.

According to recent bibliography, bizarre leiomyomas remain a controversial issue regarding the preoperative and postoperative therapeutic mapping. Giant cells with pleomorphic nuclei and little or no mitotic activity compose the microscopic analysis of such lesions.

Multidisciplinary approach is mandatory in order to establish ultimate diagnosis and treatment. Bizarre leiomyomas still represent a gray scale among the whole scientific community.
\end{abstract}

Keywords: Bizarre myomas; Laparoscopic dissection; Mirror depiction

\section{Introduction}

Incidence of uterine fibroid tumours increases as women grow older, and they may occur in more than $30 \%$ of women $40-60$ years of age [1].

Risk factors include null parity, obesity, family history, black race, and hypertension [2]. Uterine fibroids consist of smooth muscular tissue with always the possibility of malignant transformation.

Tumour size and anatomic location are strongly accompanied with assiduous therapeutic strategy. Therapeutic strategy is strongly accompanied with age and fertility capacity of the patient.

In cases of degenerated uterine fibroids in nulliparous patients, laparoscopic approach represents the gold standard of surgical confrontation. Focusing on current bibliography, classification of uterine myomas is mandatory in order to establish proper diagnosis and treatment.

Classification of uterine fibroids consists intramural, subserosal, sub mucosal and intraligamentary (inside parametrial area) [3].
Ultimate goal remains, especially in nulliparous women, extremely conservative approach, protection of fertility preservation and increase patient's quality of life.

Objective of our study reflects thorough diagnosis and assiduous treatment of clinical symptomatic and ultra-sonographic mirror depiction of two enormous intramural myomas.

\section{Case}

We present a case of a 38-year old patient (G0, P0) admitted at our Department, complaining of severe episodes of menorrhagia followed by diffuse abdominal pain.

Analysing her atomic history, she reported reception of thyroxin agents, due to her history of hypothyroidism. Undergoing of cervical cryotherapy due to infiltration of HPV (Human papilloma virus). Transvaginal ultra-sonographic evaluation depicted presence of two enormous intramural myomas maximal diameter around $7 \mathrm{~cm}$ (Figure 1). 

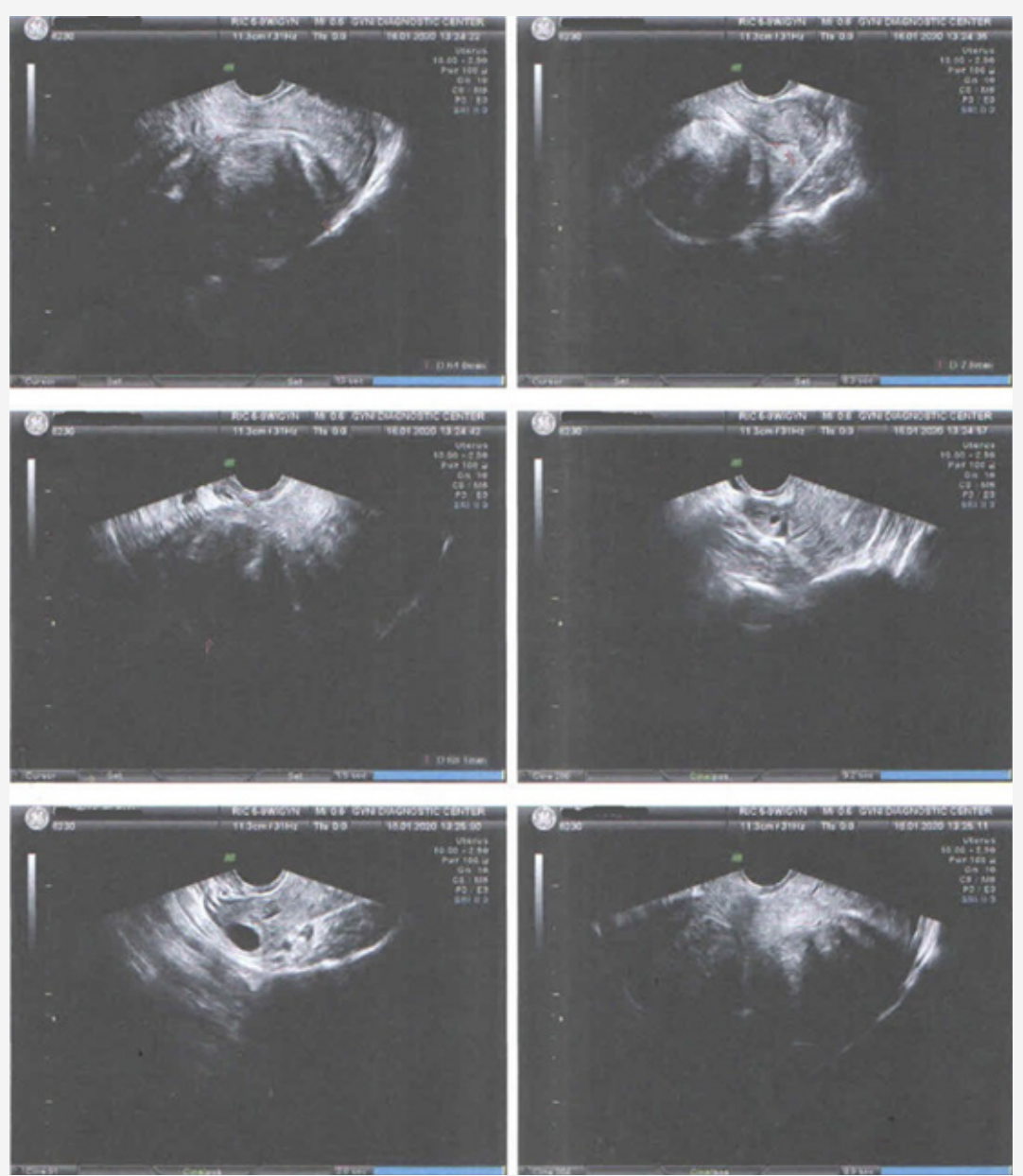

Figure 1: Transvaginal ultra-sonographic mirror-like depiction of intramural myomas.

Diagnostic curettage did not reveal signs of malignancy, detecting existence of endometrial polyp. Pap smear without areas of neoplasmatic infiltration.

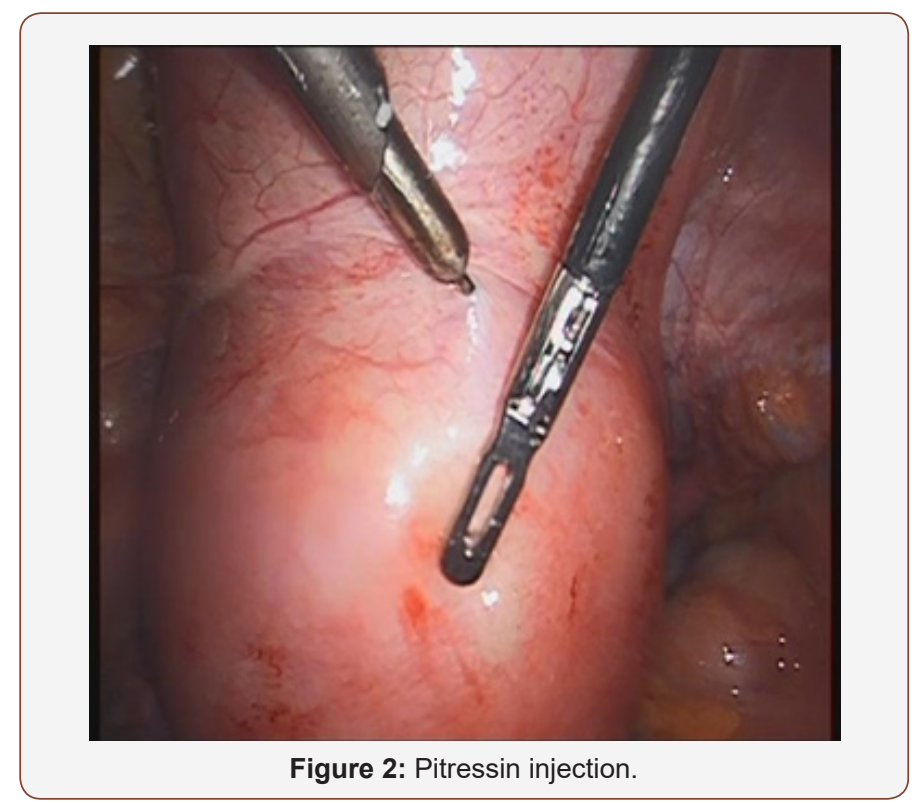

Abdominal MRI confirmed all imaging findings, consisting of conservative therapeutic mapping, focusing always on fertility preservation. Patient underwent laparoscopic dissection of intramural myomas, as standard of care towards the establishment of proper therapeutic strategy.

Preoperative injection of pitressin in order to decrease any intraoperative myometrial bleeding (Figure 2). Penetrating the abdominal cavity two enormous intramural myomas mirror shaped was performed. Same maximal diameter, origin and depth of infiltration.

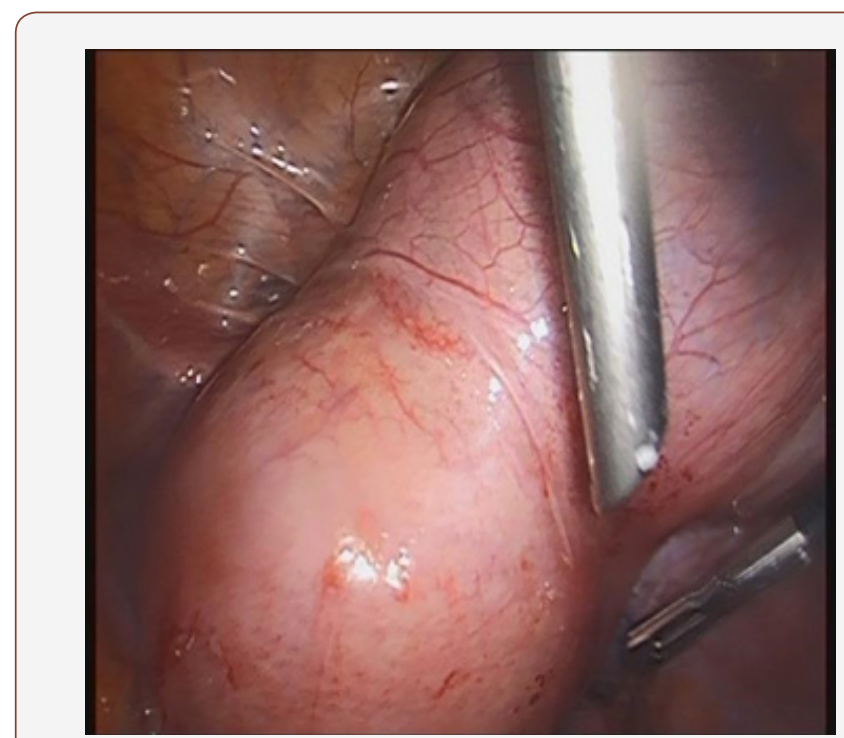

Figure 3: Mirror-like depiction of intramural myomas. 
Assiduous dissection followed by thorough haemostasis was mandatory. Histologic evaluation revealed presence of two intramural myomas maximal diameter of $7 \mathrm{~cm}$, covered with gigantic and multinuclear areas of bizarre myomas. (Figure 3).

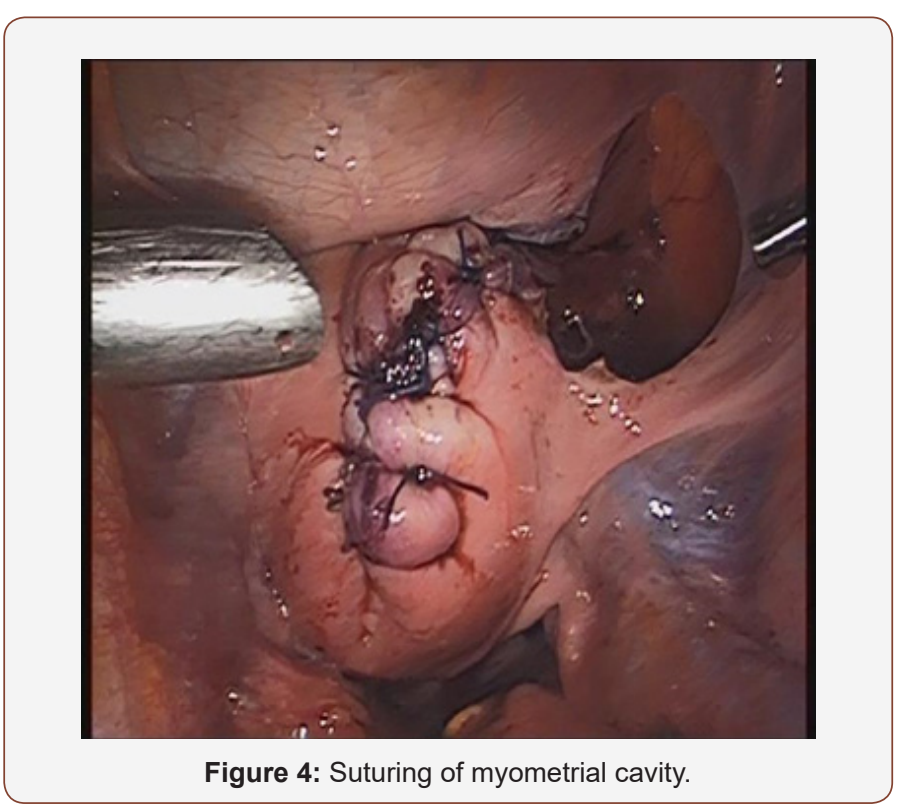

Suturing of myometrial cavity and washing of peritoneal cavity was performed (Figure 4). Last step of therapeutic mapping was morcellation of intramural myomas (Figure 5). Patient was discharged from our Department next pod in good clinical condition.

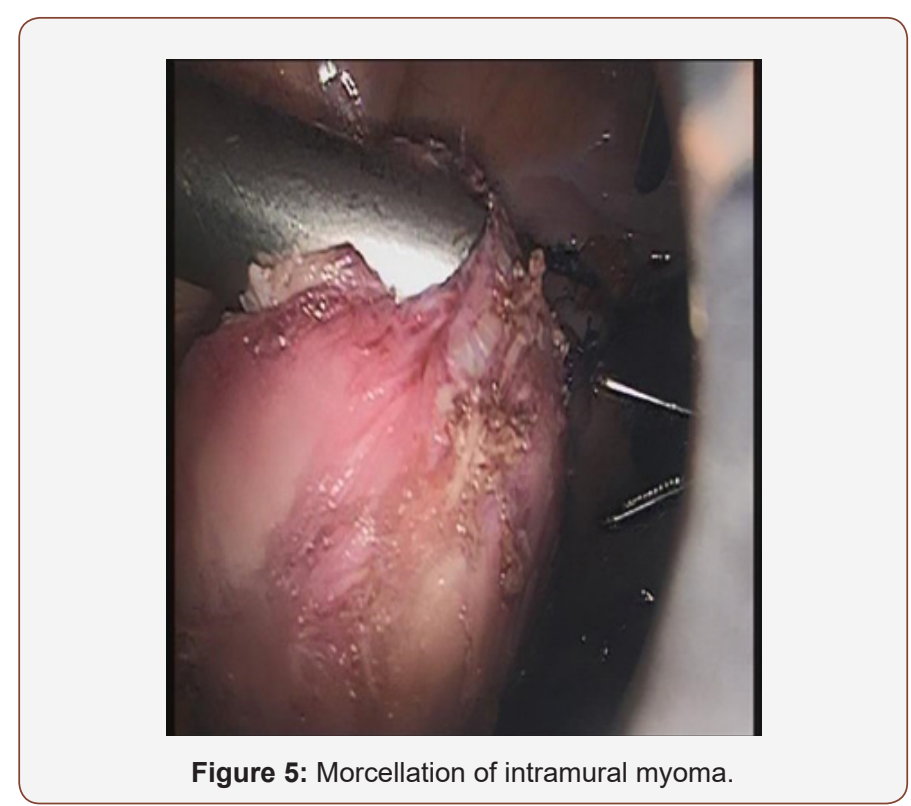

\section{Discussion}

Many factors affect the therapeutic mapping of uterine myomas. Age of the patient, gynecologic or obstetrical history, previous surgical procedures and fertility preservation.

Uterine fibroids consist of smooth muscular tissue with always the possibility of malignant transformation [4]. Tumour size and anatomic location are strongly accompanied with assiduous therapeutic strategy [5].
Transvaginal ultrasonography reflects the first preoperative procedure, depending on the physician's experience and technical sufficiency of the ultrasound machine. Imaging findings as areas of cystic degeneration, enlarged and asymmetric vascularization, papillary protrusions, and possibly increased tumour markers as Ca-125/Ca 15-3/Ca 19-9 reveal preoperative procedures of malignant metaplasia [6].

In such cases, abdominal MRI can, without a doubt, guide the preoperative management [6]. After WHO (World Health Organization) classification bizarre leiomyomas presented as fibroids with giant cells with pleomorphic nuclei and little or no mitotic activity [7].

In many cases they represent a histologic gray zone concerning the therapeutic mapping in female patients of reproductive age. Before final diagnosis is established, assiduous examination of the specimen is mandatory focusing on terms of atypia or necrosis (simple, moderate, or severe).

Along with genetic predisposition and ovarian hormone stimulation, many growth factors are identified. Besides genetic predisposition and ovarian hormones that play a major role in tumour expansion, a large number of growth factors have also been identified which favour expansion.

These are insulin-like growth factor (IGF), epidermal growth factor (EGF) and platelet-derived growth factor (PDGF), transforming growth factor beta (TGF beta), and basic fibroblast growth factor (BFGF) [8]. These may have a role to play in tumour expansion. The major differential dilemma remains the establishment of bizarre uterine myomas versus endometrial stromal sarcoma (ESS).

The main characteristics of ESS consist of infiltrative myometrium growth and vascular invasion, presence of necrotic areas, and mitotic activity [9].

Due to infiltration of the myometrial basal membrane, surgical dissection after staging of the lesion represents the gold standard. Multidisciplinary approach is mandatory in order to establish proper postoperative treatment.

In cases of metastatic ESS, neoadjuvant therapy or series of radiotherapy will under stage the tumour expansion and make the tumour staging surgically feasible. On the other hand, patients with positive progesterone or estrogen receptors (ER+, PR+), can be treated postoperatively with hormonal agents such as progestogens [10].

\section{Conclusion}

Bizarre uterine fibroids represent a controversial scientific zone in the current bibliography. More studies must be conducted in order to establish proper diagnosis and treatment. Multidisciplinary approach is mandatory in cases of patients of reproductive age. Ultimate goal remains in such cases, always fertility preservation. 


\section{Acknowledgement}

None.

\section{Conflict of Interest}

Authors declare no conflict of interest.

\section{References}

1. Bulun SE (2013) Uterine fibroids. N Eng J Med 369(14): 1344-1355.

2. Chander B, Shekhar S (2015) Osseous metaplasia in leiomyoma: A first in a uterine leiomyoma. J Cancer Res Ther 11(3): 661.

3. Laughlin-Tommaso SK, Hesley GK, Hopkins MR, Brandt KR, Zhu Y, Stewart EA (2017) Clinical limitations of the International Federation of Gynecology and Obstetrics (FIGO) classification of uterine fibroids. International Journal of Gynaecology and Obstetrics 139(2): 143-148.

4. Nakai G, Yamada T, Hamada T, Atsukawa N, Tanaka Y, et al. (2017) Pathological findings of uterine tumors preoperatively diagnosed as red degeneration of leiomyoma by MRI. Abdom Radiol (NY) 42(7): 1825-1831.
5. Solomon LA, Schimp VL, Ali-Fehmi R, Diamond MP, Munkarah AR (2005) Clinical update of smooth muscle tumors of the uterus. J Minim Invasive Gynecol 12(5): 401-408.

6. Duvnjak S, Ravn P, Green A, Andersen PE (2027) Uterine fibroid embolization with acrylamido polyvinyl microspheres: Prospective 12-month clinical and MRI follow-up study. Acta Radiolo 58(8): 952-958.

7. Downes KA, Hart WR (1997) Bizarre leiomyomas of the uterus. A comprehensive pathologic study of 24 cases with long term follow up. Ame J Surg Pathol 21(11): 1261-1270.

8. Srinivasan R, Saraiya U (2004) Recurrent fibroids. Journal of Obstetrics and Gynecology of India. 54: 363-366.

9. Yang KH, Shin JA, Jung JH, Jung HW, Lee HR, et al. A case of metastatic low-grade endometrial stromal sarcoma treated with letrozole after ovarian ablation by radiotherapy. Cancer Res Treat. 47(4): 958-962.

10. Chang KL, Grabtree GS, Lim-Tan Kempson RL, Hendrickson MR (1994) Primary uterine endometrial stromal neoplasms. A clinicopathologic study of 117 cases. The Am J Surg Pathol 14(5): 415-438. 